\title{
On L-Fuzzy $\sigma$-Generalized Closed Sets and their Properties
}

\author{
Baby Bhattacharya \\ Assistant Professor \\ Department of Mathematics, NITAgartala, \\ Barjala, Jirania-799055, Tripura, India.
}

\author{
Jayasree Chakraborty \\ Research Scholar \\ Department of Mathematics, NITAgartala, \\ Barjala, Jirania-799055, Tripura, India.
}

\begin{abstract}
The aim of this paper is to introduce the concept of fuzzy $\sigma$ generalized closed set and study its properties. Secondly the concept of infra $L$-fuzzy topological space is introduced. Making use of fuzzy $\sigma$-closed sets and fuzzy $\sigma$-generalized closed sets the concept of fuzzy $\sigma$-generalized continuous mapping is introduced. Lastly the concept of fuzzy $\sigma$ generalized closed irresolute map in $L$-fuzzy topological spaces are given.
\end{abstract}

\section{General Terms}

L-Fuzzy topological space

\section{Keywords}

coprime element, fuzzy $\sigma$-closed set, fuzzy $\sigma^{\prime}$-open set, fuzzy $\sigma$-generalized closed set, fuzzy $\sigma^{\prime}$-generalized open set.

\section{INTRODUCTION}

The concept of generalized closed set was first introduced by Levine [6] in ordinary topological space. Later on Balasubramaian and Sundaram [1] introduced the concept of generalized fuzzy closed set in fuzzy topological space. In this paper the generalization of $L$-fuzzy topological spaceis studied. In section 2 some definitions as ready references are given. Section 3 is devoted to study fuzzy $\sigma$-generalized closed sets and their properties. In this section a new space called infra $L$-fuzzy topological space also defined. In section 4 fuzzy $\sigma$-generalized continuous mapping is defined and studied its properties. Lastly the concept of fuzzy $\sigma$ generalized closed irresolute map in $L$-fuzzy topological spaces are given in section 5

Throughout this work $X$ and $Y$ will be non empty ordinary sets and $L=L(\leq, \vee, \wedge, ')$ will denote a fuzzy lattice i.e. a complete completely distributive lattice with a smallest element 0 and a largest element $1(0 \neq 1)$ and with an order-reversing involution $a \rightarrow a^{\prime}(a \in L)$. $L$ is therefore a continuous lattice. Also $L^{X}$ will denote the lattice of $L$-fuzzy subsets of $X$.

\section{PRELIMINARIES}

2.1. Definition Let $X$ be a non empty ordinary set, $L$ be a fuzzy lattice, $\delta \subset L^{X}$. $\delta$ is called an $L$-fuzzy topology on $X$, and $\left(L^{X}, \delta\right)$ is called an $L$-fuzzy topological space if $\delta$ satisfies the following conditions i) $\underline{0}, \underline{1} \in \delta$ ii)for any $A, B \in \overline{\delta, A} \wedge B \in \delta$ iii) $\forall A \subset \delta, \vee A \in \delta$

Particularly, when $L=[0,1]$, call an $L$-fuzzy topological space $\left(L^{X}, \delta\right)$ a fuzzy topological space and denote it by $(\mathrm{X}, \delta)$.
2.2 Definition [5] An element $p$ of $L$ is called prime iff $p \neq 1$ and whenever $a, b \in L$ with $a \wedge b \leq p$ then $a \leq p$ or $\quad b \leq p$. The set of all prime elements which are not 1 of $L$ will be denoted by $\operatorname{pr}(L)$.

2.3 Definition [6] Let $L$ be a fuzzy lattice. $\alpha \in L$ is called a union-irreducible (or a molecule or coprime) element of $L$, if for arbitrary $a, b \in L$, we have $\alpha \leq a \vee b \Rightarrow \alpha \leq a$ or $\alpha$ $\leq b$. The set of all nonzero union-irreducible elements of $L$ is denoted by $M(L)$. The set of all molecules for a fuzzy lattice $L^{X}$ is denoted by $M *\left(L^{X}\right)$. Clearly $p \in p r(L)$ iff $p^{g} \in M(L)$.

2.4 Definition [8] Let $\left(L^{X}, \delta\right)$ be an $L$-fuzzy topological space and $\alpha \in M(L)$ and $A \in L^{X}, A$ is called an $\alpha$-closed set , if for any $x \in X, C l A(x) \geq \alpha \Rightarrow A(x) \geq \alpha$

The set of all $\alpha$-closed set in $\left(L^{X}, \delta\right)$ is denoted by $C_{\alpha}(\delta)$. Clearly, $\forall \alpha \in M(L), \delta^{\prime} \subset C_{\alpha}(\delta)$.

2.5 Theorem Let $\left(L^{X}{ }_{\alpha} \delta\right)$ be an $L$-fts, $\alpha \in M(L), A \in L^{X}$. Then $\mathrm{A}$ is $\alpha$-closed iff $A^{p}$ is $\alpha^{x}$-open.

2.6 Definition [1] A fuzzy set $A$ in an fts $X$ is called generalized fuzzy closed (in short, gf-closed) if $\operatorname{cl}(A) \leq U$ whenever $A \leq U$ and $U$ is fuzzy open.

2.7 Definition [1] A function $f: X \rightarrow Y$ is called generalized fuzzy continuous (in short gf-continuous)if the inverse image of every fuzzy closed set in $Y$ is gf-closed in $X$.

2.8 Definition [1] A function $f: X \rightarrow Y$ is called fuzzy gc-irresolute if the inverse image of every gf-closed set in $Y$ is gf-closed in $X$.

2.9 Definition [3] Let $\left(L^{X}{ }_{0} \delta\right)$ be an L-fts, $\alpha \in M(L), \lambda \in L^{X} \quad \lambda$ is called fuzzy generalized $\alpha$-closed set,if $c l_{\alpha}(\lambda) \leq \mu$ whenever $\lambda \leq \mu$ and $\mu$ is fuzzy $\alpha^{x}$-open set.

\section{FUZZY $\sigma$-GENERALIZED CLOSED SETS IN L-FUZZY TOPOLOGICAL SPACES}

In this section, fuzzy $\sigma$-closed set, fuzzy $\sigma$-generalized closed set is defined and studied its properties.

\subsection{Definition}

Let $\left(L^{X}, \delta\right)$ be an $L$-fuzzy topological space and $\sigma \in M(L)$ and $A \in L^{X}, A$ is called a fuzzy $\sigma$-closed set, if for any $x \in X$, $\mathrm{Cl}(\operatorname{Int}(\mathrm{Cl} A(x))) \geq \sigma \Rightarrow A(x) \geq \sigma$ 
The set of all fuzzy $\sigma$-closed set in $\left(L^{X}, \delta\right)$ is denoted by $C_{\sigma}(d)$.

\subsection{Remark}

Fuzzy closed set and fuzzy $\sigma$-closed set are independent concept as seen in the following example.

\subsection{Example}

Let $X=\left\{x_{1}, x_{2}, x_{2}\right\}$. Define $f_{1 v} f_{2 v} f_{a}: X \rightarrow[0,1]$ as follows $f_{1}=0_{\mathrm{X}}, f_{2}=1_{\mathrm{X}}, f_{\mathrm{a}}=\left\{\left(x_{1}, 0.5\right),\left(x_{2}, 0.7\right),\left(x_{\mathrm{a}}, 0.6\right)\right\}$

Clearly $\delta=\left\{f_{10} f_{2}, f_{3}\right\}$ is an $L$-fts on $X$.

$\left\{\left(x_{1}, 0.5\right),\left(x_{2}, 0.3\right),\left(x_{2}, 0.4\right)\right\}$ is fuzzy closed but this is not a fuzzy $\sigma$-closed set for $\sigma=0.3$.

\subsection{Definition}

Let $\left(L^{X}, \delta\right)$ be an $L$-fuzzy topological space and $\sigma \in M(L)$ and $A \in L^{X}, A^{x}$ is called a fuzzy $\sigma^{x}$-open set iff $A$ is called an fuzzy $\sigma$-closed set.

\subsection{Definition}

If $\lambda$ is an $L$-fuzzy set in a $L$-fts $L^{X}$ and $\sigma \in M(L)$ then $\sigma l_{\sigma}(\lambda)=\cap\{\mu: \mu \geq \lambda\}, \mu$ is "fuzzy $\sigma$-closed set", is called $\sigma$ closure of $\lambda$.

An $L$-fuzzy set $\lambda$ in a $L$-fts $\left(L^{X}, \delta\right)$ is a fuzzy $\sigma$-closed iff $\lambda=$ $\mathrm{cl}_{\sigma}(\lambda)$.

\subsection{Definition}

Let $\left(L^{X}, \tilde{\delta}\right)$ be an $L$-fts, and $\sigma \in M(L)$, and $\lambda \in L^{X}$. $\lambda$ is called an fuzzy $\sigma$-generalized closed set(in short F $\sigma$-gclosed set), if $c l_{\sigma}(\lambda) \leq \mu$ whenever $\lambda \leq \mu$ and $\mu$ is fuzzy $\sigma^{x}$-open set.

\subsection{Definition}

If $\lambda_{1}$ and $\lambda_{2}$ are F $\sigma$-gclosed sets then $\lambda_{1} \vee \lambda_{2}$ is a F $\sigma$-gclosed set.

Proof: Let $\lambda_{1} \vee \lambda_{2} \leq \mu$ where $\mu$ is fuzzy $\sigma^{x}$-open set. Since $\lambda_{1}$ and $\lambda_{2}$ are F $\sigma$-gclosed sets therefore $c l_{\sigma}\left(\lambda_{1}\right) \leq \mu$ whenever $\lambda_{1} \leq \mu$ and $c l_{\sigma}\left(\lambda_{2}\right) \leq \mu$ whenever $\lambda_{2} \leq \mu$. $c l_{\sigma}\left(\lambda_{1} \vee \lambda_{2}\right)=c l_{\sigma}\left(\lambda_{1}\right) \vee c l_{\sigma}\left(\lambda_{2}\right)$ whenever $\lambda_{1} \vee \lambda_{2} \leq \mu$ and $\mu$ is fuzzy $\sigma^{\prime \prime}$-open set.

However the intersection of two F $\sigma$-gclosed sets is not $\mathrm{F} \sigma$ gclosed set as the following example shows.

\subsection{Example}

The intersection of two $\mathrm{F} \sigma$-gclosed sets is not $\mathrm{F} \sigma$-gclosed set. Let $X=\left\{x_{1}, x_{2}, x_{a}\right\}$. Define $f_{1 x} f_{2}, f_{a x} f_{4} f_{5}: X \rightarrow[0,1]$ as follows

$f_{1}=0_{X}, f_{2}=1_{X}, f_{a}=\left\{\left(x_{1}, 0.5\right),\left(x_{2}, 0.5\right),\left(x_{a}, 0.5\right)\right\}$,

$f_{4}=\left\{\left(x_{1}, 0.4\right),\left(x_{2}, 0.3\right),\left(x_{\mathrm{a}}, 0.2\right)\right\}$,

$f_{5}=\left\{\left(x_{1}, 0.7\right),\left(x_{2}, 0.6\right),\left(x_{\mathrm{g}}, 0.8\right)\right\}$

Clearly $\delta=\left\{f_{10} f_{2}, f_{3}, f_{4}, f_{5}\right\}$ is an $L$-fts on $\mathrm{X}$. Define $\lambda_{1}, \lambda_{2}: X \rightarrow[0,1]$ as follows:

$\left.\lambda_{1}=\left\{x_{1}, 0.9\right),\left(x_{2}, 0.4\right),\left(x_{2}, 0.3\right)\right\}$ and

$\lambda_{2}=\left\{\left(x_{1}, 0.5\right),\left(x_{2}, 0.4\right),\left(x_{2}, 0.9\right)\right\}$.

Here $\lambda_{1}$ and $\lambda_{2}$ are F $\sigma$-gclosed sets but $\lambda_{1} \wedge \lambda_{2}=$ $\left\{\left(x_{1}, 0.5\right),\left(x_{2}, 0.4\right),\left(x_{2}, 0.3\right)\right\}$ is not $\mathrm{F} \sigma$-gclosed set where $=\frac{1}{2}$.

\subsection{Theorem}

If $\lambda$ is $\mathrm{F} \sigma$-gclosed set and $\lambda \leq \mu \leq c l_{\sigma}(\lambda)$, then $\mu$ is $\mathrm{F} \sigma$ gclosed set .

Proof: Let $\beta$ be a fuzzy $\sigma^{x}$-open set such that $\beta \geq \mu$. Since $\mu \geq \lambda, \beta \geq \lambda$, and $\lambda$ is F $\sigma$-gclosed set,$\beta \geq c l_{\sigma}(\lambda)$. But $c l_{\sigma}(\lambda) \geq c l_{\sigma}(\mu)$. since $c l_{\sigma}(\lambda) \geq \mu$ and so $\beta \geq c l_{\sigma}(\mu)$. Hence $\mu$ is $\mathrm{F} \sigma$-gclosed set.

\subsection{Definition}

A fuzzy set $\lambda$ is called fuzzy $\sigma^{*}$-generalized open (in short $\mathrm{F} \sigma^{*}$-gopen) iff 1 - $\lambda$ is $\mathrm{F} \sigma$-gclosed.

We now prove some properties of fuzzy $\sigma^{x}$-generalized open sets.

\subsection{Remarks}

(1) The union of two $\mathrm{F} \sigma^{x}$-gopen sets is not generally $\mathrm{F} \sigma^{x}$ gopen.

Example 3.8 serves the purpose.

(2) The intersection of any two $\mathrm{F} \sigma^{x}$-gopen sets is $\mathrm{F} \sigma^{x}$-gopen.

Proof: Let $\lambda_{1}$ and $\lambda_{2}$ are $\mathrm{F} \sigma^{x}$-gopen sets in $L^{X}$ then $\lambda_{1}{ }^{x}$ and $\lambda_{2}{ }^{*}$ are F $\sigma$-gclosed set in $L^{X}$. By theorem 3.7 $\lambda_{1}{ }^{n} \vee \lambda_{2}{ }^{*}$ is F $\sigma$ gclosed. $\lambda_{1}{ }^{p} \vee \lambda_{2}{ }^{g}=\left(\lambda_{1} \wedge \lambda_{2}\right)^{s}$ is $F \sigma$-gclosed set. Therefore $\left(\lambda_{1} \wedge \lambda_{2}\right)$ is $\mathrm{F} \sigma^{x}$-gopen set.

\subsection{Definition}

If $\lambda$ is an $L$-fuzzy set in a $L$-fts $L^{X}$ and $\sigma^{\prime} \in P(L)$ then int $\sigma^{r}(\lambda)=U\{\mu: \lambda \geq \mu\}, \mu$ is fuzzy $\sigma^{x}$-open set, is called fuzzy $\sigma^{x}$-interior of $\lambda$.

An $L$-fuzzy set $\lambda$ in a $L$-fts $\left(L^{X}, \delta\right)$ is a fuzzy $\sigma^{x}$-open iff $\lambda=\operatorname{int}_{\sigma} r(\lambda)$.

\subsection{Theorem}

An $L$ - fuzzy set $\lambda$ is $\mathrm{F} \sigma^{\prime}$-gopen $\Leftrightarrow \mu \leq \operatorname{int}_{\sigma^{\prime}}(\lambda)$ whenever $\mu$ is F $\sigma$-closed set and $\mu \leq \lambda$.

Proof: Let $\lambda$ be a fuzzy $\sigma^{*}$-gopen set and $\mu$ be a F $\sigma$-closed set such that $\mu \leq \lambda$. Therefore $1-\lambda \leq 1-\mu$ and $1-\lambda$ is $\mathrm{F} \sigma$ gclosed set. $c l_{\sigma}(1-\lambda) \leq 1-\mu$.i.e $1-c l_{\sigma}(1-\lambda) \geq 1-(1-\mu)=$ $\mu$.

But $1-c l_{\sigma}(1-\lambda)=$ int $_{\sigma^{r}}(\lambda) \times$ Therefore $\mu \leq$ int $_{\sigma^{x}}(\lambda)$.

Conversely suppose that $\lambda$ is an L-fuzzy set such that $\mu \leq$ int $_{\sigma}(\lambda)$ whenever $\mu$ is $\mathrm{F} \sigma$-closed set and $\mu \leq \lambda$. We claim that $1-\lambda$ is $\mathrm{F} \sigma$-gclosed set. So $1-\lambda \leq \mu$ where $\mu$ is fuzzy $\sigma^{x}$-open. $1-\lambda \leq \mu \Rightarrow 1-\mu \leq \lambda$.

Hence by assumption we must have $1-\mu \leq$ int $_{\sigma^{x}}(\lambda)$.i.e. 1 -int $\sigma^{r}(\lambda) \leq \mu$. But $1-$ int $_{\sigma^{r}}(\lambda)=c l_{\sigma}(1-\lambda) \leq \mu$. This shows that $1-\lambda$ is $F \sigma$-gclosed set. Therefore $\lambda$ is $F \sigma^{x}$-gopen.

\subsection{Theorem}

If int $\sigma^{r}(\lambda) \leq \mu \leq \lambda$ and $\lambda$ is fuzzy $\sigma^{*}$-generalized open, then $\mu$ is fuzzy $\sigma^{x}$-generalized open.

Proof: Given int $\sigma^{r}(\lambda) \leq \mu \leq \lambda$. We have $1-\lambda \leq 1-$ $\mu \leq 1-i n \sigma_{\sigma^{r}}(\lambda)=c l_{\sigma}(1-\lambda)$. As $\lambda$ is fuzzy $\sigma^{x}$. generalized open, $1-\lambda$ is $F \sigma$-gclosed and so it follows by theorem 3.9 that is $1-\mu$ is fuzzy $\sigma$-generalized closed, i.e. $\mu$ is fuzzy $\sigma^{x}$-generalized open. 


\subsection{Definition}

Let $X$ be a non empty ordinary set, $L$ be a fuzzy lattice, $\delta \subset L^{X}$. $\delta$ is called an $L$-fuzzy infra topology on $X$, and $\left(L^{X}, \delta\right)$ is called an $L$-fuzzy infra topological space if $\delta$ satisfies the following conditions

i) $\underline{0}, \underline{1} \in \delta$

ii) for any $A, B \in \delta, A \wedge B \in \delta$

\subsection{Theorem}

Let $F G O_{\sigma^{r}}(\delta)=\left\{\right.$ The set of all $\mathrm{F} \sigma^{*}$-gopen in $\left.\left(L^{X}, \delta\right)\right\}$. Then $\left(L^{\mathrm{X}}, F G O_{\sigma^{x}}(\delta)\right)$ forms an infra L-fuzzy topological space.

Proof: It is obvious from remarks 3.11.

\subsection{Definition}

A function $f: L^{X} \rightarrow L^{Y}$ is called fuzzy $\sigma$-continuous if the inverse image of every fuzzy closed set in $L^{Y}$ is fuzzy $\sigma$-closed set in $L^{X}$.

Example. Let $f: L^{X} \rightarrow L^{Y}$ where $X=\left\{x_{1}, x_{2}, x_{2}\right\}$ and $Y$ $=\left\{y_{1}, y_{2}, y_{a}\right\}$. We define $A: X \rightarrow[0,1]$ as follows $A\left(x_{1}\right)=$ $A\left(x_{a}\right)=1, A\left(x_{2}\right)=0$. Clearly $\tau=\left\{1_{X}, 0_{X} A\right\}$ forms an L-fts on $X$. Let $Y=\left\{y_{1} v y_{2} v y_{2}\right\}, B: Y \rightarrow[0,1]$ defined as follows $B\left(y_{1}\right)=B\left(y_{2}\right)=1, B\left(y_{a}\right)=0 . \delta=\left\{1_{W^{*}} 0_{Y^{v}} B\right\}$ forms an $L$-fts on $Y$. Let $f$ is defined as $f\left(x_{1}\right)=y_{1 v} f\left(x_{2}\right)=y_{2} v f\left(x_{a}\right)=y_{a}$.

Here $f$ is fuzzy $\sigma$-continuous.

\subsection{Remarks}

$L$-fuzzy continuouse fuzzy $\sigma$-continuous

Example. (i) $f: L^{X} \rightarrow L^{Y} X=\left\{x_{1}, x_{2}, x_{a}\right\}$. We define $A$ : $X \rightarrow[0,1]$ as follows $A\left(x_{1}\right)=0.5, A\left(x_{2}\right)=0.7, A\left(x_{a}\right)=0.6$. Clearly $\tau=\left\{1_{X}, 0_{X} A\right\}$ forms an L-fts on $X$. Let $Y=$ $\left\{y_{1} y_{2} y_{b}\right\}, \quad B: Y \rightarrow[0,1]$ defined as follows $B\left(y_{1}\right)=0.7$, $B\left(y_{2}\right)=0.5, B\left(y_{a}\right)=0.6 . \delta=\left\{1_{W^{3}} 0_{Y^{v}} B\right\}$ forms an L-fts on $Y$. Here $f$ is defined as $f\left(x_{1}\right)=y_{2} v f\left(x_{2}\right)=y_{10} f\left(x_{a}\right)=y_{a}$ .Here $f$ L-fuzzy continuous but $f$ is not fuzzy $\sigma$-continuous. Since the inverse image of fuzzy closed set $\left\{\left(y_{1}, 0.3\right),\left(y_{2}\right.\right.$, $\left.0.5),\left(y_{a}, 0.4\right)\right\}$ in $L^{Y}$ is not fuzzy $\sigma$-closed set in $L^{X}$.

(ii) $f: L^{X} \rightarrow L^{Y} X=\left\{x_{1}, x_{2}, x_{2}\right\}$, Define $A: X \rightarrow[0,1]$ as follows $A\left(x_{1}\right)=1, A\left(x_{2}\right)=0, A\left(x_{a}\right)=0$. Clearly $\tau=\left\{1_{X}, 0_{X} A\right\}$ forms an L-fts on $X$. Let $Y=\left\{y_{1} y_{2} x y_{a}\right\}, \quad B$ : $Y \rightarrow[0,1]$ defined as follows $B\left(y_{1}\right)=1, B\left(y_{2}\right)=1, B\left(y_{a}\right)=0$. $\delta=\left\{1_{Y^{s}} 0_{Y} B\right\}$ forms an L-fts on $Y$. Let $f$ is defined as $f\left(x_{1}\right)=y_{1 v} f\left(x_{2}\right)=y_{a v} f\left(x_{2}\right)=y_{2}$. Here $f$ is fuzzy $\sigma$ continuous but not $L$-fuzzy continuous. Since the inverse image of fuzzy closed set $\left\{\left(y_{1}, 0\right),\left(y_{2}, 0\right),\left(y_{a}, 1\right)\right\}$ in $L^{Y}$ is not fuzzy closed set in $L^{X}$ but it is fuzzy $\sigma$-closed in $L^{X}$.

\subsection{Definition}

A map $f: L^{X} \rightarrow L^{Y}$ is called fuzzy $\sigma$-closed (in short $\mathrm{F} \sigma$ closed) set if the image of every fuzzy $\sigma$-closed set in $L^{X}$ is fuzzy $\sigma$-closed in $L^{Y}$.

\subsection{Theorem}

If $\lambda$ is $\mathrm{F} \sigma$-gclosed set in $L^{X}$ and if $f: L^{X} \rightarrow L^{Y}$ is fuzzy $\sigma$ continuous and F $\sigma$-closed, then $f(\lambda)$ is F $\sigma$-gclosed set in $L^{Y}$.
Proof: If $f(\lambda) \leq \mu$ where $\mu$ is fuzzy open in $L^{Y}$, then $\lambda \leq f^{-1}(\mu)$. Since $\lambda$ is F $\sigma$-gclosed and $f^{-1}(\mu)$ is fuzzy $\sigma^{x}$ open, $c l_{\sigma}(\lambda)=\bar{\lambda} \quad \leq f^{-1}(\mu)$. i.e. $f(\bar{\lambda}) \leq \mu$. Now by assumption, $f(\bar{\lambda}) \quad$ is $\quad$ F $\sigma$-closed $\overline{f(\lambda)} \leq \overline{f(\bar{\lambda})}=f(\bar{\lambda}) \leq \mu$. We know that $\overline{f(\lambda)}=c l_{\sigma}(f(\lambda))$. Thuscl $l_{\sigma}(f(\lambda)) \leq \mu$. This means $f(\lambda)$ is F $\sigma$-gclosed set in $L^{Y}$.

\subsection{Example}

Under F $\sigma$-closed, fuzzy $\sigma$-continuous maps fuzzy $\sigma^{*}$ generalized open sets are generally not taken into fuzzy $\sigma^{x}$ generalized open sets.

Let $X=\{a\}, Y=\{a, b, c\}, \tau_{1}=\left\{0_{X^{s}} 1_{X}\right\}, \tau_{2}=\left\{0_{W^{s}} 1_{Y^{s}} B_{1}\right\}$, $B_{1}, Y \rightarrow[0,1]$ is such that $B_{1}(a)=B_{1}(c)=1, B_{1}(b)=0$. Clearly $\tau_{1}$ and $\tau_{2}$ are $L$-fuzzy topologies on $X$ and $Y$ respectively.

Define $f: L^{X} \rightarrow L^{Y}$ as follows $f(a)=b$.

One can verify $f$ is $f \sigma$-continuous and $\mathrm{F} \sigma$-closed.Now we shall show that $f$ does not take fuzzy $\sigma^{x}$-generalized open sets to fuzzy $\sigma^{x}$-generalized open. Clearly $1_{X}$ is fuzzy $\sigma^{x}$ generalized open in $L^{X}$ but $f\left(1_{X}\right)=\left\{\left(a_{v}, 0\right)\left(b_{v} 1\right),\left(c_{v}, 0\right)\right\}$ is not fuzzy $\sigma^{x}$-generalized open in $L^{Y}$.

\section{FUZZY -GENERALIZED CONTINUOUS MAPPING AND ITS PROPERTIES}

\subsection{Definition}

A map $f: L^{X} \rightarrow L^{Y}$ is called fuzzy $\sigma$-generalized continuous (in short $\mathrm{F} \sigma$-gcontinuous) if the inverse image of every fuzzy closed set in $L^{Y}$ is $\mathrm{F} \sigma$-gclosed in $L^{X}$.

Some properties of fuzzy $\sigma$-generalized continuous function has given.

\subsection{Theorem}

If $f: L^{X} \rightarrow L^{Y}$ is fuzzy $\sigma$-continuous then it is fuzzy $\sigma$ generalized continuous but the converse is not true.

\subsection{Example}

Let $X=\left\{x_{1 v} x_{2}, x_{a}\right\}, Y=\left\{y_{1 v} y_{2 v} y_{a}\right\}, \quad, \quad \tau_{1}=\left\{0_{X} 1_{X}\right\}$, $\tau_{2}=\left\{0_{W^{*}} 1_{Y_{v}} B_{1}\right\}, \quad B_{1}: Y \rightarrow[0,1]$ is such that $B\left(y_{1}\right)=1$, $B\left(y_{2}\right)=1, B\left(y_{2}\right)=0$. Clearly $\tau_{1}$ and $\tau_{2}$ are $L$-fuzzy topologies on $X$ and $Y$ respectively. Define $f: L^{X} \rightarrow L^{Y}$ as follows $f\left(x_{1}\right)=y_{1} v f\left(x_{2}\right)=y_{a} v f\left(x_{2}\right)=y_{2}$. Here $f$ is fuzzy $\sigma$ generalized continuous but not fuzzy $\sigma$-continuous.

\subsection{Theorem}

Let $f: L^{X} \rightarrow L^{Y}$ be a function,

The following statements are equivalent

(i) $f$ is fuzzy $\sigma$-generalized continuous

(ii) The inverse image of each fuzzy open set in $L^{Y}$ is $\mathrm{F} \sigma^{x}$ gopen in $L^{X}$.

Balasubramanian and Sundaram [1] defined the generalized fuzzy closure operator $\mathrm{cl}^{*}$ to obtain some properties of $g$-continuity. So, in similarway, we define the 
fuzzy $\sigma$-generalized closure operator $\mathrm{cl}^{*}$ for any L-fuzzy set A in $\left(L^{X}, \tau\right)$ as follows:

$\mathrm{cl}^{*}(\lambda)=\AA\left\{\mu_{i} \mu \geq \lambda\right.$ and $\lambda$ is $\mathrm{F} \sigma$-gclosed $\}$.

\subsection{Theorem}

Let $f: L^{X} \rightarrow L^{Y}$ be a fuzzy $\sigma$-generalized continuous, then $f(\operatorname{cl} *(\lambda)) \leq \operatorname{cl}(f(\lambda))$ where $\lambda$ is any fuzzy set in $L^{X}$.

Proof: Let $\lambda$ be any fuzzy set in $L^{X}$. Then $\lambda \leq f^{-1}(f(\lambda)) \leq f^{-1}(c l(f(\lambda)))$. Now $\operatorname{cl}(f(\lambda))$ is a fuzzy closed in $L^{Y}$. As $f$ is fuzzy $\sigma$-generalized continuous, $f^{-1}\left(\operatorname{cl}(f(\lambda))\right.$ is fuzzy $\sigma$-generalized in $L^{X}$. $\mathrm{cl}^{*}(\lambda) \leq f^{-1}(\operatorname{cl}(f(\lambda))$. Hence $f(\operatorname{cl} *(\lambda)) \leq \operatorname{cl}(f(\lambda))$.

\section{FUZZY - -gc-IRRESOLUTE FUNCTIONS AND THEIR PROPERTIES}

\subsection{Definition}

A function $f: L^{X} \rightarrow L^{Y}$ is called fuzzy $\sigma$-gc irresolute if the inverse image of every $\mathrm{F} \sigma$-gclosed set in $L^{Y}$ is $\mathrm{F} \sigma$-gclosed in $\mathrm{L}^{x}$.

Following are the properties of fuzzy $\sigma$-gc irresolute maps.

\subsection{Theorem}

If $f: L^{X} \rightarrow L^{Y}$ is fuzzy $\sigma$-gc irresolute if the inverse image of every $\mathrm{F} \sigma^{x}$-gopen set in $L^{Y}$ is $\mathrm{F} \sigma^{x}$-gopen in $L^{X}$.

\subsection{Remarks}

Fuzzy $\sigma$-gc irresolute fuzzy $\sigma$-generalized continuous

\subsection{Example}

(i) Let $X=\left\{x_{1}, x_{2}, x_{2}\right\}, Y=\left\{y_{1} v y_{2} v y_{a}\right\},, \tau_{1}=\left\{0_{X} 1_{X} A\right\}$, $\tau_{2}=\left\{0_{F}, 1_{Y}\right\}, A: X \rightarrow[0,1]$ is such that $A\left(x_{1}\right)=0, A\left(x_{2}\right)=$ $1, A\left(x_{2}\right)=0$. Clearly $\tau_{1}$ and $\tau_{2}$ are $L$-fuzzy topologies on $X$ and $Y$ respectively. Define $f: L^{X} \rightarrow L^{Y}$ as follows $f\left(x_{1}\right)=y_{2} f\left(x_{2}\right)=y_{1} f\left(x_{a}\right)=y_{a}$. Here $f$ is fuzzy $\sigma$-generalized continuous but not fuzzy $\sigma$-gc irresolute. Since $\left\{\left(y_{1}, 1\right),\left(y_{2}, 0\right),\left(y_{y}, 1\right)\right\}$ is $\mathrm{F} \sigma$-gclosed set in $L^{Y}$ but its inverse image is not $\mathrm{F} \sigma$-gclosed set in $\mathbb{L}^{X}$.

(ii) Let $X=\left\{x_{1}, x_{2}, x_{a}\right\}, Y=\left\{y_{1} v y_{2} v y_{a}\right\}, \quad, \tau_{1}=\left\{0_{X^{s}} 1_{X^{v}} A\right\}$, $\tau_{2}=\left\{0_{Y^{s}} 1_{Y^{v}} B\right\}, A, B: X \rightarrow[0,1]$ is such that $A\left(x_{1}\right)=0.7$, $A\left(x_{2}\right)=0.5, A\left(x_{a}\right)=0.6 ; B\left(x_{1}\right)=0.5, B\left(x_{2}\right)=0.7, B\left(x_{a}\right)=$ 0.6 . Clearly $\tau_{1}$ and $\tau_{2}$ are $L$-fuzzy topologies on $X$ and $Y$ respectively. Define $f: L^{X} \rightarrow L^{Y}$ as follows $f\left(x_{1}\right)=y_{2} v f\left(x_{2}\right)=y_{1 v} f\left(x_{a}\right)=y_{a}$. Here $f$ is fuzzy $\sigma$-gc irresolute but not fuzzy $\sigma$-generalized continuous.

\subsection{Theorem}

Letf: $\left(L^{X}{ }_{v} \tau\right) \rightarrow\left(L^{Y}{ }_{x} \delta\right)$ and $g:\left(L^{Y}{ }_{s} \delta\right) \rightarrow\left(L^{Z}{ }_{x} \eta\right)$ be any two functions. Then

(i) $g \circ f$ is fuzzy $\sigma$-generalized continuous, if $g$ is fuzzy $\sigma$ generalized continuous and $f$ is fuzzy $\sigma$-gc irresolute.

(ii) $g \circ f$ is fuzzy $\sigma$-gc irresolute, if $g$ is fuzzy $\sigma$-gc irresolute and $f$ is fuzzy $\sigma$-gc irresolute.
Proof: (i) Let $F$ be a fuzzy closed in $\left(L^{Z}{ }_{x} \eta\right)$. Then $g^{-1}(F)$ is fuzzy $\sigma$-generalized closed in $\left(L^{Y}{ }_{g} \delta\right)$. Since $g$ is fuzzy $\sigma$ generalized continuous. $f^{-1}\left(g^{-1}(F)\right)=(g \circ f)^{-1}(F)$ is fuzzy $\sigma$-generalized closed $\operatorname{in}\left(L^{X}{ }^{x} \tau\right)$. Since $f$ is fuzzy $\sigma$-gc irresolute. Hence $g \circ f$ is fuzzy $\sigma$-generalized continuous.

(ii) Let $F$ be a fuzzy $\sigma$-generalized closed in $\left(L^{Z}{ }^{Z} \eta\right)$. Then $g^{-1}(F)$ is fuzzy $\sigma$-generalized closed in $\left(L^{Y}{ }_{x} \delta\right)$. Since $g$ fuzzy $\sigma$-gc irresolute. $f^{-1}\left(g^{-1}(F)\right)=(g \circ f)^{-1}(F)$ is fuzzy $\sigma$-generalized closed in $\left(L^{X}{ }_{\sigma} \tau\right)$. Since $f$ is fuzzy $\sigma$-gc irresolute. Hence $g \circ f$ is fuzzy $\sigma$-gc irresolute.

\subsection{Remarks}

The composition of two fuzzy $\sigma$-generalized continuous need not be fuzzy $\sigma$-generalized continuous function.

\section{CONCLUSION}

The concept of infra L-fuzzy topological space is introduced with the help of the properties of F $\sigma^{x}$-gopen sets. There is a scope to study the properties of infra L-fuzzy topological space and its application. There is a future scope to study Compactness, connectedness and separation axioms in infra L-fuzzy topological space.

\section{ACKNOWLEDGEMENTS}

The authors would like to thank the referee for the valuable comment and suggestion.

\section{REFERENCES}

[1] Balasubramanian.G,Sundaram.P, On some generalization of fuzzy continuous functions, Fuzzy Sets and Systems 86(1997)93-100.

[2] Chang.C.L,Fuzzy topological spaces, J.Math.Anal.Appl.24 (1968)182-190.

[3] Daraby Bayaz and Nimse.S.B, On Fuzzy Generalized $\alpha$ closed set and its applications, Faculty of Sciences and Mathematics, University of Nis, Serbia (2007),99-108.

[4] Dunham.W, A new closure operator for non $T_{1}$ topologies, Math.J.22(1982)55-60.

[5] Gierz.G. et al, A compendium of Continuous Lattice Springer, Berlin, 1980.

[6] Gujon Wang, Theory of topological molecular lattices, Fuzzy Sets and Systems 47 (1992) 351-376.

[7] Levine. N, Generalized closed sets in topology, Rend, Circ.Mat.Palermo 19(1970)89-96.

[8] Yongming. Li, The theory of L-fuzzy closed graph and strong closed graph, Fuzzy SystemsMath. 2 (1991) 3037. 